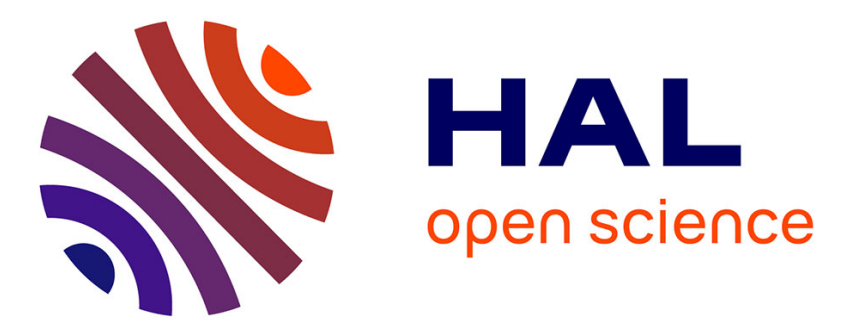

\title{
Crime et famines. Europe, Afrique, Asie, XIXe et XXe siècles
}

François Bonnet

\section{To cite this version:}

François Bonnet. Crime et famines. Europe, Afrique, Asie, XIXe et XXe siècles. Déviance et Société, 2021, Varia, 45 (4), pp.645-662. 10.3917/ds.454.0131 . halshs-03472992

\section{HAL Id: halshs-03472992 \\ https://shs.hal.science/halshs-03472992}

Submitted on 10 Dec 2021

HAL is a multi-disciplinary open access archive for the deposit and dissemination of scientific research documents, whether they are published or not. The documents may come from teaching and research institutions in France or abroad, or from public or private research centers.
L'archive ouverte pluridisciplinaire HAL, est destinée au dépôt et à la diffusion de documents scientifiques de niveau recherche, publiés ou non, émanant des établissements d'enseignement et de recherche français ou étrangers, des laboratoires publics ou privés. 


\title{
Crime et famines. Europe, Afrique, Asie, $19^{\mathrm{e}}$ et $20^{\mathrm{e}}$ siècles
}

\author{
François Bonnet \\ CNRS - UMR Pacte, Sciences Po Grenoble, Université Grenoble Alpes \\ francois.bonnet@umrpacte.fr
}

UMR Pacte, Institut d'études politiques

BP 48, 38040 Grenoble cedex 9

France

\section{Résumé.}

Cette revue de littérature porte sur l'effet de la famine sur la criminalité et vise à démontrer que la pauvreté absolue est criminogène. Les travaux issus de la recherche en histoire dans des pays et à des époques différentes (Europe, Afrique, Asie, $19^{\mathrm{e}}$ et $20^{\mathrm{e}}$ siècles) suggèrent une augmentation des vols et le développement d'une petite délinquance de nécessité lors des famines, avec notamment une plus grande participation des femmes. Les approches économétriques identifient un effet causal de l'augmentation des prix alimentaires sur les atteintes à la propriété en analysant notamment l'effet des variations climatiques ou celui des parasites agricoles. Les deux approches, historiques et économétriques, tendent à constater que la faim n'entraîne pas une augmentation des violences criminelles. L'augmentation des vols pendant les famines n'est vraisemblablement donc pas liée à un surcroît d'activité du système pénal.

\section{Abstract.}

This literature review focuses on the effect of famine on crime and aims to demonstrate that absolute poverty is criminogenic. Historical research in different countries and eras (Europe, Africa, Asia, $19^{\text {th }}$ and $20^{\text {th }}$ centuries) suggest an increase in thefts and the development of a delinquency of necessity during famines, with greater participation of women. Econometric approaches identify a causal effect of the increase in food prices on property crime by analyzing the effect of climatic variations or that of agricultural parasites. The two approaches, historical and econometric, tend to note that hunger does not result in an increase in criminal violence. The increase in thefts during famines is thus probably not linked to an increase in criminal system.

Mots-clés: crime, famine, faim, prix alimentaires

Keywords: crime, famine, hunger, food prices 
Dans son ouvrage de référence Famine : A Short History, l'historien irlandais Cormac Ó Gráda consacre le chapitre "Les horreurs de la famine » au crime, à l'esclavage, à la prostitution, aux infanticides et abandons d'enfants, et au cannibalisme. La présente revue de littérature propose de revenir sur l'effet de la famine sur le crime.

On retient pour cet article la définition du crime des sciences sociales, c'est-à-dire en ignorant les distinctions du droit français entre crime, délinquance et contraventions, et en la restreignant aux vols et aux violences, qui sont les types de faits les plus constamment réprouvés dans le temps et dans l'espace. On évacuera du périmètre de cet article les débats autour de la qualification des famines elles-mêmes comme des «crimes», ou du comportement des autorités politiques durant les famines comme des actes criminels (de Waal, 1997). On évoquera brièvement la question de ceux qui profitent des famines, qui est une question importante mais périphérique à l'objet de cet article, lequel est l'effet de la pauvreté absolue sur la propension aux vols et aux violences. C'est pourquoi on s'intéressera à deux types de phénomènes : l'effet des famines proprement dites, avec une surmortalité importante, et tel que documenté par la recherche historique ; et l'effet des fortes tensions sur les prix alimentaires dans les sociétés souvent rurales qui ne disposent pas d'un système de protection sociale, et qui se prête à l'analyse de séries de données notamment au moyen des approches économétriques.

Poser la question de l'effet de la famine sur la criminalité revient à se focaliser sur un caslimite d'une question plus grande, qui est celle de la relation entre pauvreté (absolue) et crime $^{1}$. Cette relation est plus compliquée à établir qu'il n'y paraît, et est controversée en criminologie. Du côté des criminologies positivistes, les enquêtes par autorévélation des années 1960 alimentent les explications psychologiques qui font de l'impulsivité le déterminant central du crime (Hirschi, 1969). Une méta-analyse influente montre à la fin des années 1970 un trend historique de moindre importance de la classe sociale comme facteur explicatif du crime (Tittle, Villemez et Smith, 1978). L'augmentation de la criminalité aux Etats-Unis dans les années 1960-1970 conduit à théoriser que le crime est en fait corrélé à la prospérité (Cohen et Felson, 1979). Les enquêtes longitudinales des années 1980 nourrissent les étiologies du crime en termes de déficit familial (Loeber \& Stouthamer-Loeber, 1986). Dans les années 1990, les travaux sur le capital social dominent le champ (Sampson, Raudenbush et Earls, 1997). Du côté des criminologies critiques, on s'est plutôt intéressé aux phénomènes de criminalisation (notamment à partir de Becker, 1963) et à la problématisation des institutions pénales (notamment à partir de Foucault, 1975); les tentatives de vérifier empiriquement les théories marxistes de la criminalité (Rusche et Kirchheimer, 1939) ont produit des résultats mitigés (Chiricos et Delone, 1992). Montrer l'effet de la faim sur le crime n'est donc pas un résultat trivial, d'une part parce que la littérature notamment francophone sur les famines ne s'est pas attardée sur le sujet, et d'autre part parce que la criminologie a presque ignoré les famines.

L'article étudie donc l'effet de la famine sur le crime, en prenant soin de dissocier les atteintes à la propriété des violences, et en commençant par documenter l'augmentation des vols dans la recherche historique. La plus grande participation des femmes à la criminalité pendant les famines suggère que les famines déclenchent, de manière bien compréhensible, une délinquance de nécessité. L'article rend ensuite compte des travaux reposant sur les techniques économétriques et qui portent non pas sur les famines stricto sensu, mais sur l'augmentation des prix alimentaires dans des sociétés agraires (et ce pour des raisons d'accès

\footnotetext{
${ }^{1}$ Il existe également une littérature conséquente sur la corrélation entre inégalités (et non pauvreté) et violence (Blau et Blau, 1982 ; Messner et Rosenfeld, 1997 ; Fajnzylber et al. 2002).
} 
aux données ${ }^{1}$ ). Les tensions sur les prix alimentaires déclenchent également une augmentation de la délinquance acquisitive, ce qui montre un effet causal de la pauvreté absolue sur le crime. L'augmentation des vols pendant les famines n'est vraisemblablement pas liée à un surcroît d'activité du système pénal, puisque les violences (criminelles), au contraire, n'augmentent pas.

\section{Famines et vols de nécessité dans la recherche historique}

Il existe de nombreux exemples historiques d'augmentation des vols durant une famine. Les exemples les mieux documentés sont ceux des famines du XIX ${ }^{\mathrm{e}}$ siècle en Europe du Nord. Entre 1845 et 1850, les famines liées au mildiou de la pomme de la terre tuent des dizaines de milliers de personnes dans les Flandres belges et en Russie, et culminent dans la Grande Famine irlandaise. En Irlande, les cambriolages sont multipliés par 5, et de nombreux voleurs arrêtés meurent de faim et de maladie en prison (Ó Gráda, 2009, p. 53). En Belgique flamande, entre 1840 et 1847 , lorsque les prix alimentaires sont au plus haut, les incriminations pour mendicité sont multipliées par 9, les vagabondages et les petits vols par 3 (Vanhaute, 2007). Vingt ans après, en Finlande, de mauvaises récoltes en 1865 et 1867 provoquent une grave crise alimentaire qui tue plus de 150.000 Finlandais entre 1866 et 1868 . Alors que les taux de criminalité finlandais sont tendanciellement baissiers au cours du XIX siècle, le taux d'atteintes à la propriété (les vols) est soudainement multiplié par 3,5 (Vuorela, 2017).

En dehors de l'Europe, pendant les famines de 1866, 1873-74 et 1896-97 au Bengale, on constate également une hausse des vols (Mukherjee 1993). D'ailleurs, les administrateurs britanniques ont bien compris le mécanisme, puisque les hausses de la criminalité sont analysées comme des signes avant-coureurs d'une famine dans les Indian Famine Codes, qui sont une échelle de la gravité des famines conçue par les autorités coloniales britanniques pour anticiper et gérer les pénuries alimentaires (Arnold, 1979 ; Ó Gráda, 2009 ; Papaionnou, 2017). Lors de la famine en Inde de 1876-1878, les femmes et les enfants qui meurent de faim sont empêchés de voler ou de glaner par « le marquage au fer, la torture, la mutilation du nez, et étaient parfois tués » (Davis, 2002, p. 46). En Afrique, au Burundi, les greniers collectifs prescrits par les autorités coloniales ne sont finalement acceptés par la population que lors de la famine de 1943-1944, parce qu'ils permettent notamment de limiter les vols (Feltz et Bidou, 1994).

Les famines dans les pays communistes au $\mathrm{XX}^{\mathrm{e}}$ siècle ne font pas exception. Pendant la famine qui fait 5 millions de morts durant la guerre civile en Russie (1921-1922), les petits vols et chapardages sont constants, de même que pendant le siège de Leningrad (1941-1943), où les malades sont apparemment volés sur leur lit d'hôpital (rapporté par Ó Gráda, 2009). Durant la famine en Chine provoquée par le Grand Bond en avant en 1959-1961, un nombre non-négligeable du total des morts (15 à 36 millions) serait causé par des tabassages à mort faisant suite à des suspicions de vols (Ó Gráda, 2013, citant l'historienne sino-britannique Xun Shou), par exemple en cachant les décès au sein de la famille afin de continuer à toucher les coupons alimentaires (Dikötter, 2010).

Plusieurs éléments suggèrent que cette augmentation des vols en période de rareté alimentaire est directement liée à la nécessité. Lors de la famine irlandaise, la taille moyenne des

\footnotetext{
${ }^{1}$ Les travaux qui reposent sur des approches économétriques nécessitent des données fiables sur l'évolution de nombreux indicateurs, ce qui empêche l'étude de sociétés en guerre, lesquelles sont souvent la cause de la famine.
} 
prisonniers à Dublin augmente, ce qui signifie que les gens d'un statut social plus élevé se mettent à commettre des crimes, probablement poussés par la faim (Ó Gráda, 2009). Pendant la famine en Finlande de 1866-1868, la valeur moyenne des biens volés baisse de moitié, suggérant la multiplication des petits vols de survie (Vuorela, 2017). Lors d'une pénurie de carburant à Madagascar en 2002, les vols dans les champs (une forme de glanage) augmentent, tandis que les vols de bétail, qui sont la prérogative du crime organisé, ne varient pas pendant l'épisode de pauvreté aggravée (Fafchamps et Minten, 2006).

Du point de vue des dynamiques de genre, on sait que les hommes commettent structurellement la grosse majorité des actes de délinquance dans toutes les sociétés ; or, les famines font augmenter la criminalité des femmes. Dans les Flandres belges, les condamnations pour prostitution sont multipliées par 7 pendant la famine, et le profil général des délinquants évolue, devenant plus jeune et plus féminin (Vanhaute, 2007). La corrélation constatée entre les prix du blé et le taux de criminalité en France au XIX ${ }^{\mathrm{e}}$ siècle est encore plus forte chez les femmes que chez les hommes : les variations des prix du blé expliquent $50 \%$ de la variance du taux de criminalité des hommes, contre $65 \%$ pour celui des femmes (Gouda et Smith, 1983). Le fait que les hausses de prix du blé fassent relativement plus voler les femmes que les hommes accrédite l'idée de vols de nécessité.

Les petits vols de nécessité ne doivent pas occulter les gros vols de prédation commis par les différentes entités en capacité de le faire. Les famines occasionnent d'importantes restructurations foncières. Ainsi, après la famine irlandaise, le nombre de petits propriétaires de moins de 2 hectares est divisé par deux, tandis que le nombre de propriétaires de plus de 12 hectares est multiplié par trois (cité dans Brunel, 2002). On peut analyser ce phénomène comme relevant pour une part de la prédation, comme un abus de faiblesse : la faim pousse les tous petits propriétaires (des paysans pauvres) à vendre à vil prix leurs terres pour pouvoir s'acheter de la nourriture devenue hors de prix. Ces paysans pauvres vendent tous en même temps à ceux qui peuvent acheter, c'est-à-dire les grands propriétaires terriens qui eux ne souffrent pas de la faim, et qui profitent de l'effondrement du prix des terres pour agrandir leur domaine. Dans le cas de la famine chinoise de 1959-1961, les entreprises chinoises, théoriquement contrôlées par le Parti Communiste Chinois, se livrent à tout un répertoire de pratiques visant à s'approprier de la nourriture pour le compte des cadres du parti. Les entreprises empruntent frauduleusement à la banque d'État pour financer des festins, et se livrent à un gigantesque troc, échangeant du ciment, du béton, du charbon, du cuivre, des pneus, tout ce qui est produit contre de la nourriture, achetant parfois en gros pour spéculer sur les pénuries futures, tandis que des faux coupons alimentaires sortent par rouleaux des imprimeries d'État (Dikötter, 2010). On rentre là dans une autre dimension de la criminalité, qui n'est pas moins intéressante, mais qui ne relève pas de la problématique de l'effet de la pauvreté sur le crime.

Enfin, l'effet des famines sur les vols a également été repéré dans le cadre des migrations qui font suite aux épisodes de famine. Les vagabonds du XVI siècle jetés sur la route par la faim lors des épisodes de quasi-famines dans l'Angleterre élisabéthaine pouvaient errer plusieurs mois après la fin de la disette, se livrant à des petits larcins pour survivre loin de chez eux, alors que la situation générale était revenue à la normale (Lawson, 1986). Un effet similaire est apparemment identifiable dans les données sur la criminalité à New York dans les années 1850 , avec une très forte implication des immigrés de la Grande Famine irlandaise dans les statistiques locales - en 1858, les immigrés irlandais forment les trois quarts des admissions en prison (Ernst, 1994). En Mauritanie, les autorités de Nouakchott associent l'arrivée de 
Touaregs maliens qui fuient la famine au Sahel dans les années 1970 à un surcroît de vagabondage, de prostitution et de délinquance juvénile (Bonnecase, 2010).

On dispose donc de tout un ensemble d'éléments issus de la recherche historique qui suggère que les famines entraînent une augmentation des vols. Pour aller plus loin dans l'identification causale, il faut déplacer le champ des investigations vers la recherche en économie, qui s'intéresse non pas à la question des famines stricto sensu, mais à celle de l'effet de des variations des prix alimentaires, en particulier dans les sociétés agraires.

\section{Prix alimentaires et crime : l'identification causale par les techniques économétriques}

Les travaux qui utilisent des techniques économétriques cherchent à identifier des relations de causalité. Parmi les multiples conceptions de la causalité qui ont cours dans la philosophie et les sciences sociales, ces travaux reposent sur une conception probabiliste de la causalité : la faim cause le crime au sens où elle augmente la probabilité de comportement criminel, par opposition à une conception déterministe (où la totalité des gens qui auraient faim se mettraient à voler). Selon cette conception probabiliste, le fait d'identifier un effet de la faim sur le crime ne signifie évidemment pas que seule la faim a un effet sur le crime. En l'occurrence, montrer un effet causal, c'est montrer que la corrélation entre la faim et le crime n'est pas une coïncidence ou l'effet d'une variable confondante, mais bien que la faim entraîne le crime.

Le problème de l'identification causale de la faim sur le crime est très simple : il est probable que la faim ait un effet causal sur la criminalité, mais il est également connu qu'un contexte de criminalité élevée aggrave les difficultés de chacun, tout comme il est plausible que des variables extérieures aient à la fois un effet sur la faim et sur la criminalité. Pour isoler un effet causal, on peut utiliser (entre autres) des variables instrumentales, une technique statistique où l'on introduit dans le modèle de régression une variable pour laquelle on est à peu près sûr qu'elle ait un effet causal sur la variable indépendante (la faim) et dont on est également sûr qu'elle n'est affectée ni par la variable indépendante, ni par la variable dépendante (le crime).

La méthode des variables instrumentales n'est pas exempte de critiques. Elle est plus sujette au p-hacking (le «triturage des données » afin d'obtenir un résultat statistiquement significatif) que d'autres méthodes comme les essais randomisés controllés ou les régressions sur discontinuités; elle est aussi moins sujette au p-hacking que les régressions linéaires traditionnellement utilisées en sociologie (Brodeur et al., 2020). En particulier, le fait d'utiliser les données pluviométriques comme instrument est sujet à caution, dans la mesure où le climat a un effet sur tellement de variables qu'il est en fait peu plausible qu'il ne soit pas en interaction complexe avec d'autres variables qui interagissent avec la prix des denrées alimentaires et la criminalité (Mellon, 2021). Ces précautions n'invalident pas les résultats qui suivent, mais sont à garder à l'esprit.

Dans la mesure où les prix alimentaires sont directement liés au climat, plusieurs articles utilisant une approche économétrique reposent sur des données pluviométriques pour établir la relation causale entre les prix alimentaires et la criminalité, dans des contextes différents : en Allemagne au XIX ${ }^{\mathrm{e}}$ siècle, en Afrique au début du $\mathrm{XX}^{\mathrm{e}}$ siècle, en Inde au cours du $\mathrm{XX}^{\mathrm{e}}$ siècle, et aux Philippines contemporaines. Ces articles peuvent être distingués en deux 
catégories : ceux qui cherchent à démontrer l'effet causal de la pauvreté sur la criminalité, et ceux qui cherchent à montrer l'effet du climat sur la criminalité.

La première approche consiste donc à montrer que l'extrême pauvreté - la menace de la faim - cause un surcroît de criminalité. Au XIX ${ }^{\mathrm{e}}$ siècle, dans les pays du nord de l'Europe, la céréale de base de la population pauvre est le seigle, une céréale plus résistante au froid que le blé, mais moins riche en protéines. Le prix du seigle détermine donc dans une large mesure le niveau de vie des pauvres. Quand le prix du seigle augmente, le nombre des atteintes à la propriété augmente aussi (Mehlum et al. 2006). Pour isoler l'effet causal du prix du seigle sur la délinquance (pour montrer que la corrélation n'est pas accidentelle), Halvor Mehlum et al. utilisent les variations des précipitations en Bavière entre 1835 et 1861. La pluie a un effet causal évident sur le prix du seigle, mais les variations du crime ne font pas pleuvoir. L'effet des précipitations sur le prix du seigle permet de donc d'isoler l'effet causal spécifique du prix du seigle sur la criminalité, en dehors de tout effet que la criminalité pourrait avoir sur le prix du seigle, et indépendamment de l'effet que d'autres facteurs pourraient avoir à la fois sur le prix du seigle ou la criminalité. Christian Traxler et Carsten Burhop (2010) confirment ce résultat avec une stratégie de recherche fondamentalement similaire (données climatiques, prix du seigle, vols), mais en travaillant sur la Prusse des années 1882-1910. Kostadis J. Papaioannou (2017) fait le même raisonnement pour les colonies britanniques en Inde et Asie du sud-est au début du XX $\mathrm{X}^{\mathrm{e}}$ siècle, entre 1910 et 1940. Il montre que la quantité de riz produite est inversement corrélée aux atteintes à la propriété en utilisant les données pluviométriques pour isoler l'effet causal de la production de riz.

La démarche par les variables instrumentales permet une estimation de la direction et du degré des liens causaux qui est plus précise que les régressions linéaires traditionnelle. Ainsi, dans le cas du prix du seigle en Bavière, Mehlum et al. (2006) montrent qu'une régression linéaire ordinaire (aux moindres carrés) surestime du double l'effet de la pauvreté sur la criminalité. Papaionnou (2017) montre au contraire dans le cas de l'Inde à la fin du $\mathrm{XX}^{\mathrm{e}}$ siècle que la régression linéaire sous-estime cet effet.

Le propos de la seconde approche n'est pas, comme les travaux cités ci-dessus, de montrer l'effet des prix alimentaires ou de l'insécurité alimentaire sur le crime, mais de contribuer à une littérature émergente sur l'effet du changement climatique sur les conflits en général, dont la criminalité.

Kostadis Papaioannou et Michiel de Haas (2017), avec des données sur plusieurs pays d'Afrique subsaharienne pour les années 1920-1939, constatent une corrélation entre les anomalies climatiques (sécheresses et inondations) et la hausse des taux d'incarcération. Ils montrent que l'effet du climat passe par la perte de revenu agricole, laquelle entraine un ensemble de problèmes sociaux : pas seulement un surcroît de criminalité, mais aussi une baisse des revenus fiscaux, des problèmes de dette, voire de l'instabilité politique. Avec des données sur l'Inde des années 1971-2000, David S. Blakeslee et Ram Fishman (2015) montrent également que les précipitations anormales réduisent les revenus agricoles, ce qui augmente les taux de criminalité, particulièrement les atteintes à la propriété. La relation est stable sur la période. Enfin, Jesse K. Anttila-Hugues et Emily Wetherley (2018) analysent la relation entre climat et crime aux Philippines entre 1990 et 2018, et montrent que le mécanisme qui explique le lien causal entre les anomalies climatiques et l'augmentation des atteintes à la propriété est le faible rendement des terres agricoles. 
In fine les deux démarches, celle qui veut montrer l'effet du climat et celle qui veut montrer l'effet de la pauvreté, reconstituent la même chaîne causale: les variations climatiques affectent la production agricole, et une production agricole insuffisante dans une économie pré-industrielle entraîne une baisse des niveaux de vie qui est partiellement compensée par un surcroît d'activité criminelle, généralement des atteintes à la propriété. La cohérence de ces deux approches, qui montrent la même chose avec des objectifs différents, est de nature à accréditer le résultat.

D'autres travaux procèdent d'une stratégie de recherche similaire, mais en utilisant comme variable instrumentale les données sur la diffusion des parasites agricoles plutôt que des données pluviométriques. Ils confirment également le lien entre pauvreté et criminalité.

Le phylloxéra est un puceron qui attaque les racines de la vigne. Entre 1863 et 1890, le phylloxéra détruit petit à petit $40 \%$ des vignobles français. La production baisse, sans que le prix du vin n'augmente assez pour compenser les pertes des vignerons, et les vignes pourries ne sont pas remplacées par une autre culture. En conséquence, c'est tout un monde économique qui s'effondre, provoquant dans plusieurs départements des faillites bancaires. Le puceron se répand en France à une vitesse d'environ 30 kilomètres par an. En dévastant les vignes, le puceron a un effet causal direct sur la pauvreté ; mais la pauvreté, elle, n'a aucun effet causal sur le puceron, qui est simplement porté par les vents. Vincent Bignon et al. (2015) utilisent donc la diffusion du phylloxéra dans le temps et dans l'espace pour isoler l'effet causal d'une baisse brutale des revenus sur la criminalité. Ils montrent que les atteintes à la propriété, en particulier les vols, augmentent fortement avec le développement de la pauvreté liée au phylloxéra, et que ce résultat n'est pas lié à une autre cause comme l'évolution du système pénal ou la répartition des forces de l'ordre.

Christopher Muller et Daniel Schrage (2019) obtiennent des résultats similaires avec le cas du charançon du cotonnier (boll weevil). Il s'agit d'un petit insecte qui mange la fleur et les fibres du coton. Il apparaît au Venezuela en 1849 et se répand en Géorgie entre 1915 et 1920. Le charançon du cotonnier fait perdre leur travail à des milliers d'ouvriers agricoles et de métayers, qui sont pour la plupart noirs - en 1940, plus de 30\% des jeunes hommes noirs sont encore employés dans l'agriculture. Le nombre d'Africains-Américains qui va en prison pour des atteintes à la propriété augmente de plus d'un tiers (Muller et Schrage, 2019). L'effet est maximal dans les comtés qui dépendent le plus de la production de coton. Comme le phylloxéra, le charançon permet d'isoler l'effet causal de la pauvreté sur les vols : dans les deux cas, la destruction exogène de l'activité économique principale d'une région précarise l'ensemble de la population (l'exploitant fait faillite, les ouvriers agricoles sont au chômage, les services qui dépendent de l'économie productive périclitent), et fait augmenter les vols. Les cas de la vigne et du coton montrent que l'effet criminogène de la baisse du revenu (en l'occurrence) agricole advient quel que soit le type de culture, vivrière ou commerciale.

Enfin, des travaux sur la piraterie maritime contemporaine montrent le lien toujours présent entre appauvrissement et propension au crime. $80 \%$ de tous les biens qui sont transportés dans le monde le sont par la mer, et plus de $40 \%$ de tous les pays côtiers sont concernés par des phénomènes de piraterie. La plupart des attaques sont des expéditions mal équipées et près des côtes, la moitié échouent et $70 \%$ se passent sans violence (Flückiger et Ludwig, 2015). A partir d'une base de données sur les attaques de pirates dans 109 pays entre 2004 et 2009, Matthias Flückiger et Markus Ludwig (2015) montrent que la piraterie est causalement liée à la quantité de poisson disponible pour la pèche : conformément à ce que l'on sait par ailleurs, les pirates sont des pêcheurs qui ne trouvent plus de poisson et qui se livrent à la piraterie par 
nécessité économique de générer du revenu pour manger. La particularité du travail de $\mathrm{M}$. Flückiger et M. Ludwig est d'utiliser des données satellitaires sur l'abondance de plancton pour démontrer l'effet de la rareté des ressources halieutiques sur l'entrée dans la piraterie. Moins il y a de plancton dans l'océan, moins il y a de poissons à pécher, et plus il y a de pirates, avec une logique de substitution. Sebastian Axbard (2016) montre la même chose en se focalisant sur le cas de l'Indonésie, ce qui lui permet d'avoir des données plus précises sur le stock de poissons et sur les salaires des métiers autour de la pèche.

\section{Les violences et la faim}

Les recherches historiques et économétriques ont donc établi la relation entre la pauvreté absolue - la faim - et l'augmentation des atteintes à la propriété. Mais quand est-il des violences?

Un mot d'abord sur la qualité des statistiques criminelles. D'une manière générale, les statistiques criminelles sont doublement mauvaises, parce qu'elles sont des statistiques d'activité - et reflètent parfois plus une capacité administrative qu'une évolution effective des niveaux de la criminalité - et en raison de tous les biais imaginables dans l'enregistrement et le traitement de données éminemment sensibles. Cela dit, on considère généralement que plus les faits sont graves, mieux ils sont enregistrés. Il faut être prudent avec les statistiques des petits vols, tandis que les statistiques des homicides sont assez fiables, à part dans certains contextes et notamment dans le cas des pays où les forces de l'ordre commettent un nombre important d'homicides ${ }^{1}$. Cela implique que les résultats sur les violences (en particulier les données sur les homicides) sont a priori plus fiables que les résultats sur les atteintes envers les biens.

Il ne semble pas que les famines (avec des dizaines de milliers de morts) s'accompagnent d'une augmentation des violences, que ce soit au Bengale au XIX ${ }^{\mathrm{e}}$ siècle (Mukherjee, 1993), dans les Flandres pendant la crise du mildiou (Vanhaute, 2007) ou en Finlande en 1866-1868 (Vuorela, 2017). Eric Vanhaute constate même une baisse des violences dans les statistiques flamandes. Miikka Vuorela, dans le cas finlandais, estime que la stabilité des violences est probablement une illusion statistique, mais il n'explique pas pourquoi le même appareil statistique enregistre une explosion des vols et ne détecterait pas une hausse des violences, qui sont moins nombreuses, plus spectaculaires, et qui font plus probablement l'objet de plainte. Le cas irlandais est plus épineux, et il semble que les violences augmentent, mais pas autant que les vols, tandis que les viols baisseraient significativement (Ó Gráda, 2009). A Madras au $\mathrm{XIX}^{\mathrm{e}}$ siècle, les famines font émerger un banditisme qui se livre à des pillages, avec des effets d'hystérèse (les bandits restent des bandits après la famine), mais les homicides n'augmentent pas (Arnold, 1979).

La littérature économétrique, qui ne porte pas sur les famines mais plutôt sur les situations où des prix alimentaires élevés pénalisent très fortement des populations qui ne bénéficient pas d'un état-providence, révèle une relative convergence de résultats factuels sur l'absence d'augmentation des violences, que ce soit en Asie du sud-est au début du $\mathrm{XX}^{\mathrm{e}}$ siècle (Papaionnou, 2017), dans le cas du charançon du cotonnier en Géorgie au début du XX $\mathrm{X}^{\mathrm{e}}$ siècle

\footnotetext{
${ }^{1}$ Par exemple, les statistiques publiées par le FBI aux Etats-Unis au début des années 2010 faisaient état d'environ 400 homicides commis par des policiers par an ; ce chiffre a rapidement été réévalué à plus de 900 par an après que les émeutes de Ferguson liées à la mort de Michael Brown attirent l'attention de la presse américaine. Au Brésil, le nombre de personnes tuées tous les ans par la police au milieu des années 2010 est estimé entre 2000 et 5000 - l'ampleur de la fourchette indique la faible qualité des données officielles.
} 
(Muller et Schrage, 2019) ainsi que celui du phylloxéra en France (Bignon et al., 2015), à Madagascar pendant la crise politique de 2002 (Fafchamps et Minten, 2006) et pour les Philippines contemporaines (Anttila-Hugues et Wetherley 2018). D. Blakeslee et R. Fishman (2015), avec le cas de l'Inde entre 1970 et 2000, ainsi que Papaioannou et de Haas (2017) pour des pays d'Afrique sub-saharienne au début $\mathrm{du} \mathrm{XX}^{\mathrm{e}}$ siècle, constatent cependant une augmentation des violences, mais moindre que celle des atteintes à la propriété. En ce qui concerne les cas de la Bavière et de la Prusse au XIX siècle, Mehlum et al. (2006), et Traxler et Burhop (2010) montrent que c'est le renchérissement des prix de l'alcool, en l'occurrence de la bière, qui induit une plus faible consommation, laquelle se traduit par une moindre occurrence des violences.

L'augmentation des prix alimentaires semble donc avoir un effet plus sur les vols que sur les violences, mais pas toujours. Ainsi le cas des meurtres de sorcières en Tanzanie contemporaine. A partir d'une étude sur 67 villages ruraux tanzaniens entre 1992 et 2002, Edward Miguel (2005) montre que les épisodes de quasi-famine conduisent au doublement des meurtres de femmes âgées, généralement massacrées par leur famille à la machette. Les autorités publiques sont réticentes à intervenir sur la question, de peur d'être accusées ellesaussi de sorcellerie. Dans le folklore tanzanien, les sorcières sont censées être responsables à la fois des épidémies et des anomalies climatiques (sécheresses, inondations). Mais E. Miguel constate que les variations des meurtres de femmes âgées ne sont liées qu'aux anomalies climatiques, lesquelles, contrairement aux épidémies, occasionnent des disettes voire des famines. Ainsi, E. Miguel montre que les meurtres de sorcières sont causalement liés à l'appauvrissement matériel plutôt qu'à des phénomènes de boucs-émissaires à la suite de désastres. Son travail est une contribution à la littérature sur le phénomène qui consiste à accélérer la mortalité des personnes âgées quand la nourriture manque.

Une autre conséquence de l'augmentation des prix alimentaires sur l'ordre public est l'apparition d'émeutes de la faim. Les émeutes de la faim ne sont pas des phénomènes criminels mais peuvent être criminalisées par le pouvoir en place. La recherche en histoire a souligné le rôle joué par les émeutes de la faim lors de la Révolution française (Rudé 1964) ou la Révolution russe (Wade 2000). Walton et Seddon (1994) ont montré comment, dans les années 1970, les émeutes de la faim étaient réapparues à la faveur des politiques d'ajustement structurel dans les pays en voie de développement. Avec des données sur l'ensemble des pays du monde pour les années 1990-2011, Bellemare (2015) montre que c'est l'augmentation des prix alimentaires et non leur volatilité qui cause les émeutes. Selon Berazneva et Lee (2013), qui étudient les différents pays africains suite à la crise alimentaire mondiale de 2007-2008, les émeutes sont corrélées à la pauvreté et à la rareté de la nourriture, ainsi qu'à l'urbanisation et au caractère autoritaire du régime politique.

La relation entre crime et famines ne se réduit pas à l'effet direct de la faim sur la propension à la criminalité. Ainsi, en 1944, la Wehrmacht bloque le transport des denrées alimentaires vers les Pays-Bas. 20000 Néerlandais meurent de faim, particulièrement dans les grandes villes de l'ouest. Vingt ans plus tard, les hommes nés pendant la famine arrivent à l'âge du service militaire, et passent les examens psychiatriques standards. Les hommes dont la mère a le plus souffert de la faim pendant les deux premiers trimestres de la grossesse ont 2,5 fois plus de chances de développer des troubles psychiatriques dits de la personnalité antisociale, lesquels sont associés à une plus grande propension à la violence (Neugebauer et al., 1999).

Enfin, un mot sur un type particulier de violence, le cannibalisme, qui est souvent mentionné dans la littérature. Le sujet mérite un traitement spécifique mais le cannibalisme en période de 
famines reste un phénomène rare (sinon les gens ne mourraient pas de faim, mais d'assassinat), et concerne essentiellement le fait d'aller déterrer des cadavres pour manger les morts (Ó Gráda, 2015).

\section{Conclusion}

En mobilisant à la fois les études de cas issues de la recherche historique et les travaux économétriques focalisés sur l'identification causale, on peut raisonnablement considérer que la faim entraîne, entre autres, une augmentation des vols, mais seulement occasionnellement des violences criminelles. Cet effet est avant tout repéré dans les économies non-industrielles, en l'absence d'un état-providence qui pourrait amortir les chocs économiques. La relation entre famine et crime permet de poser un cas-limite à la discussion de l'effet de la pauvreté sur la criminalité, et montre que la faim pousse bien à la délinquance.

Aujourd'hui, la question crime / famine renvoie à deux questions. La première a trait au réchauffement climatique et à ses conséquences sur la sécurité alimentaire. La seconde porte sur l'effet différentiel des famines en milieu rural et en milieu urbain. La plupart des études mentionnées dans cet article portent sur des sociétés agraires avec une économie de subsistance. Certains travaux cités suggèrent un effet criminel des famines plus fort dans les villes. Or, les sociétés contemporaines sont de plus en plus urbaines et les populations déconnectées des lieux de production de nourriture. On peut donc s'interroger sur les effets spécifiques de la famine en milieu urbain dans les contextes les plus marqués par le réchauffement climatique.

\section{Bibliographie}

ANTTILA-HUGHES J.K., WETHERLEY E., 2016, «Climate of discontent: weather, typhoons, and crime in the Philippines, 1990-2008», Association of Environmental and Resource Economist at the Allied Social Science Associations, San Francisco.

ARNOLD D., 1979, « Dacoity and rural crime in Madras, 1860-1940 », Journal of Peasant Studies, 6(2), p. 140-167.

AXBARD S., 2016, «Income Opportunities and Sea Piracy in Indonesia: Evidence from Satellite Data », American Economic Journal: Applied Economics, 8(2), p. 154-94.

BECKER H.S., 1963, Outsiders, Glencoe, The Free Press.

BELLEMARE, M. F., 2015, «Rising food prices, food price volatility, and social unrest », American Journal of Agricultural Economics, 97(1), p. 1-21.

BERAZNEVA, J., Lee, D. R., 2013, « Explaining the African food riots of 2007-2008: An empirical analysis », Food Policy, 39, p. 28-39.

BIGNON V., CAROLI E., GALBIATI R., 2016, «Stealing to survive? Crime and income shocks in nineteenth century France », Economic Journal, 127(599), p. 19-49.

BLAKESLEE D.S., FISHMAN, R., 2018, «Weather shocks, agriculture, and crime evidence from India », Journal of Human Resources, 53(3), p. 750-782. 
BLAU, J. R., \& BLAU, P. M. 1982. «The cost of inequality: Metropolitan structure and violent crime ». American sociological review, 114-129.

BONNECASE V., 2010, «Retour sur la famine au Sahel du début des années 1970: la construction d'un savoir de crise », Politique africaine, (3), p. 23-42.

BRODEUR A., COOK N., HEYES A., 2020. « Methods Matter: P-Hacking and Publication Bias in Causal Analysis in Economics. » American Economic Review, 110(11), p. 3634-60.

BRUNEL S., 2002, Famines et politique, Paris, Presses de Sciences po.

CHIRICOS T.G., DELONE, M.A, 1992, «Labor surplus and punishment: A review and assessment of theory and evidence », Social Problems, 39(4), p. 421-446.

COHEN, L.E., FELSON, M.s, 1979, « Social change and crime rate trends: A routine activity approach », American Sociological Review, 44 (4), p. 588-608.

DAVIS M., 2002, Late Victorian holocausts: El Niño famines and the making of the Third World, Londres, Verso Books.

DE WAAL, A., 1997, Famine crimes: politics \& the disaster relief industry in Africa, Bloomington, Indiana University Press.

DIKÖTTER F., 2010, Mao's great famine: The history of China's most devastating catastrophe, 1958-1962, New York, Bloomsbury Publishing USA.

ERNST R., 1994, Immigrant Life in New York City, 1825-1863, Syracuse, Syracuse University Press.

FAFCHAMPS M., MINTEN B., 2006, « Crime, transitory poverty, and isolation: Evidence from Madagascar », Economic Development and Cultural Change, 54(3), p. 579-603.

FAJNZYLBER, P., LEDERMAN, D., \& LOAYZA, N. 2002. "Inequality and violent crime ». Journal of Law and Economics, 45(1), 1-39.

FELTZ G., BIDOU J.-E., 1994, «La famine manori au Burundi 1943-1944 ». Outre-Mers. Revue d'histoire, 81(304), p. 265-304.

FLÜCKIGER M., LUDWIG M., 2015, «Economic shocks in the fisheries sector and maritime piracy », Journal of Development Economics, 114, p. 107-125.

FOUCAULT M., 1975, Surveiller et punir, Paris, Gallimard.

GOUDA F., SMITH P.H., 1983, «Famine, Crime, and Gender in Nineteenth-century France: Explorations in Time-series Analysis », Historical Methods: A Journal of Quantitative and Interdisciplinary History, 16(2), p. 59-73.

HIRSCHI T., 1969, Causes of Delinquency, Berkeley, University of California Press. 
LAWSON P., 1986, «Property crime and hard times in England, 1559-1624 », Law and History Review, 4(1), p. 95-127.

LOEBER R., STOUTHAMER-LOEBER M., 1986, «Family factors as correlates and predictors of juvenile conduct problems and delinquency », Crime and Justice, 7, p. 29-149.

MEHLUM H., MIGUEL E., TORVIK R., 2006, «Poverty and crime in 19th century Germany », Journal of Urban Economics, 59(3), p. 370-388.

MELLON J., 2020. «Rain, Rain, Go Away: 137 Potential Exclusion-Restriction Violations for Studies Using Weather as an Instrumental Variable. »SSRN Electronic Journal, doi: $\underline{10.2139 / \mathrm{ssrn} .3715610 .}$.

MESSNER, S. F., ROSENFELD, R. 1997. «Political restraint of the market and levels of criminal homicide: A cross-national application of institutional-anomie theory ». Social Forces, 75(4), 1393-1416.

MIGUEL E., 2005, «Poverty and witch killing », Review of Economic Studies, 72(4), p. 1153-1172.

MUKHERJEE A., 1993, «Scarcity and crime: A study of 19th Century Bengal », Economic and Political Weekly, 28 (6), p. 237-243.

MULLER C., SCHRAGE D., 2019, «The Political economy of Incarceration in the US South, 1910-1925 : Evidence from a Shock to Tenancy and Sharecropping », IRLE Working Paper No. 105-19, http://irle.berkeley.edu/files/2019/09/The-Political-Economy-ofIncarceration-in-the-US-South-1910-1925.pdf

NEUGEBAUER R., HOEK H.W., SUSSER E., 1999, «Prenatal exposure to wartime famine and development of antisocial personality disorder in early adulthood », Journal of the American Medical Association, 282(5), p. 455-462.

Ó GRADA C., 2009, Famine: A short history, Princeton, Princeton University Press.

Ó GRADA C., 2013, «Great Leap, Great Famine: A Review Essay », Population and Development Review, 39 (2), p. 333-346.

Ó GRADA C., 2015, Eating People is Wrong, and Other Essays on Famine, its Past, and its Future, Princeton, Princeton University Press.

PAPAIOANNOU K.J., 2017, " "Hunger makes a thief of any man": Poverty and crime in British colonial Asia ». European Review of Economic History, 21(1), p. 1-28.

PAPAIOANNOU K.J., DE HAAS M., 2017, «Weather shocks and agricultural commercialization in colonial tropical Africa: did cash crops alleviate social distress?». World Development, 94, p. 346-365.

RUDÉ, G., 1964, The Crowd in History, New York, Wiley. 
RUSCHE G., KIRCHHEIMER O., 1939, Peine et structure sociale. Histoire et théorie critique du régime pénal. Paris, Cerf, 1994.

SAMPSON R.J., RAUDENBUSH S.W., EARLS F.. 1997, « Neighborhoods and violent crime: A multilevel study of collective efficacy ». Science, 277(5328), p. 918-924.

TITTLE C.R., VILLEMEZ W.J., SMITH D.A. 1978, «The myth of social class and criminality: An empirical assessment of the empirical evidence ». American Sociological Review, p. 643-656.

TRAXLER C., BURHOP C., 2010, «Poverty and crime in 19th century Germany: A reassessment », MPI Collective Goods Preprint, (2010/35).

VANHAUTE E., 2007, " "So worthy an example to Ireland". The subsistence and industrial crisis of 1845-1850 in Flanders », When the Potato Failed. Causes and Effects of the Last European Subsistence Crisis. 1845-1850, C. O Grada, R. Paping, E. Vanhaute, éd., Turnhout, Brepols, p. 123-148.

VUORELA M., 2017, "Criminality and the Finnish Famine of 1866-1868 », In A. G. NEWBY (ed.) The Enormous Failure of Nature: Famine and Society in the Nineteenth Century, COLLeGIUM, 22, p. 119-150.

WADE, R. A., 2000, The Russian Revolution, 1917. Cambridge, Cambridge University Press.

WALTON, J. K., SEDDON, D., 1994, Free Markets and Food Riots: The Politics of Global Adjustment, New York, Wiley. 